\title{
Análisis microestructural y diseño de cigüeñal y biela de un motor mono cilíndrico de 4 tiempos
}

\section{Microstructural analysis and crankshaft and connecting rod design of a 4- stroke cylindrical mono engine}

Celin Padilla Padilla. ${ }^{1}$, Bolivar Cuaical Angulo. ${ }^{2} \&$ Luis Buenaño Moyano. ${ }^{3}$

\begin{abstract}
.
DOI: https://doi.org/10.33262/cienciadigital.v3i1.293

In this project, the crankshaft and connecting rod of a four-stroke engine of a motorcycle are characterized. We analyzed the efforts to which the crankshaft connecting rod assembly is subjected by Finite Element Methods. In addition, the characterization of the connecting rod and crankshaft was carried out through microstructure tests, with which the material of each element was specified. The results obtained from the tests of the material that makes up the crankshaft - connecting rod assembly are the AISI 5120 and AISI 1050 steels, said materials do not exist in the country because they are exclusive steels for these elements, so it is recommended to use AISI steel 1045 and AISI 304 steel because their mechanical and chemical properties are similar. In the thermodynamic calculation it was determined that: the minimum force is $257.71 \mathrm{~N}$, the maximum force is $16128.23 \mathrm{~N}$ and the effective average force is $6402.01 \mathrm{~N}$. Later these forces are used in the analysis for obtaining maximum and minimum effort, these being 1.10x108 $\mathrm{Pa}$ and $57.69 \mathrm{~Pa}$ respectively, with a maximum safety coefficient of 15 and a minimum of 3.36 .
\end{abstract}

Keywords: Crankshaft, Connecting Rod, Crankshaft-Connecting Rod Set, Motorcycle.

\section{Resumen.}

\footnotetext{
${ }^{1}$ Escuela Superior Politécnica de Chimborazo, Facultad de Mecánica, Chimborazo, Ecuador, c_padilla@espoch.edu.ec

${ }^{2}$ Escuela Superior Politécnica de Chimborazo, Facultad de Mecánica, Chimborazo, Ecuador, bcuaical@espoch.edu.ec

${ }^{3}$ Escuela Superior Politécnica de Chimborazo, Facultad de Mecánica, Chimborazo, Ecuador, lfbuenanio@espoch.edu.ec
} 
En este proyecto se caracteriza el cigüeñal y la biela de un motor de cuatro tiempos de una motocicleta. Se analizó los esfuerzos a los que está sometido el conjunto cigüeñal - biela mediante Métodos de Elementos Finitos. Además, se realizó la caracterización de la biela y cigüeñal mediante pruebas de microestructura, con lo que se especificó el material de cada uno los elementos. Los resultados obtenidos de las pruebas del material que conforma el conjunto cigüeñal - biela son los aceros AISI 5120 y AISI 1050, dichos materiales no existen en el país debido a que son aceros exclusivos para estos elementos, por lo que se recomienda utilizar el acero AISI 1045 y el acero AISI 304 debido a que sus propiedades mecánicas y químicas son similares. En el cálculo termodinámico se determinó que: la fuerza mínima es de 257,71 N, la fuerza máxima es de $16128,23 \mathrm{~N}$ y la fuerza media efectiva es de $6402,01 \mathrm{~N}$. Posteriormente estas fuerzas son utilizadas en el análisis para la obtención del esfuerzo máximo y mínimo siendo estos de 1,10x108 $\mathrm{Pa}$ y 57,69 $\mathrm{Pa}$ respectivamente, con un coeficiente de seguridad máximo de 15 y mínimo de 3,36

Palabras claves: Cigüeñal, Biela, Conjunto Biela Manivela, Motocicleta

\section{Introducción.}

En los últimos años se ha realizado un impulso al desarrollo de la matriz productiva en diferentes ámbitos de la producción, en donde se pide la creación de partes y piezas en gran cantidad. Por lo que es relevante la caracterización y diseño de diferentes partes de diferentes vehículos y motocucletas. En nuestro país se están ensamblando motocicletas, pero no se está implementando elementos de producción nacional, debido a la falta de recursos económicos, conocimiento, inversionistas, etc. (Villena Izurieta, 2015)

Los elementos que conforman el conjunto biela-manivela como es el cigüeñal, la biela y la muñequilla, estos elementos están sometidos a cargas de compresión, tracción, flexión, y torsión por lo que el material del que están construidos debe estar diseñadas para resistir dichas cargas a altas revoluciones. Una de las primeras acciones que se debe hacer para poder producir partes y piezas automotrices en nuestro medio es la de partir de los elementos que ya están diseñados, y realizar una ingeniería inversa, la cual está encaminado al estudio, diseño y caracterización, específicamente del cigüeñal y la biela de un motor de 4 tiempos y 125 cc de una motocicleta. (Rovira \& Muñoz, 2015)

\section{Metodología}

La metodología de este estudio se basó en la aplicación de la ingeniería inversa, por medio del desarrollo del estado de arte se determinó el funcionamiento y la interacción de cada componente que conforma el conjunto cigüeñal - biela. (Hernández Sampieri, Fernández Collado, \& Baptista Lucio, 2010) 
Se realizó in modelado y diseño del conjunto cigüeñal - biela, ya que se debe realizar un análisis de esfuerzos, se utilizó un software CAD en el que se puede operar a través de una interfaz gráfica y generar un modelo en tres dimensiones de gran precisión. (SolidWorks Corporation, 2017)

Para el modelado del cigüeñal se tomó en cuenta los contrapesos ya que estos no son iguales debido a que cumplen diferentes funciones. Este elemento está formado por un piñón para la transmisión del movimiento hacia la cadena, en cambio el contrapeso que tienen un acople para la muñequilla y el eje esta acoplada con un rodamiento que evita vibraciones hacia la cadena. (Fuentes, 2011)

Figura 1. Contrapesos del cigüeñal
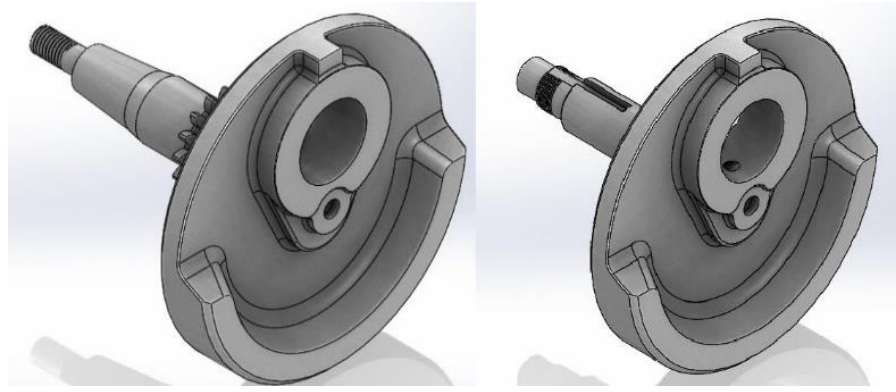

Para el modelado de la biela, se determina que es de perfil doble T que evita el pandeo que se puede provocar las fuerzas de tracción y compresión que está sometido este elemento. Además, se debe tener en cuenta los orificios de lubricación con el bulón del pistón. El pie de biela se acopla a la muñequilla formando el conjunto cigüeñal - biela. (Barbieri, Bruno, Luchi, \& Rotini, 2009)

Figura 2. Biela

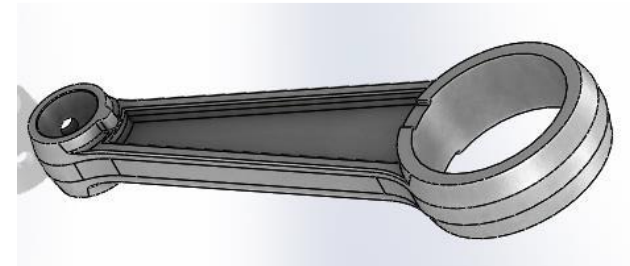

En el diseño de la muñequilla del cigüeñal, se dibujó de forma cilíndrica para facilitar la transformación del movimiento rectilíneo a giratorio de todo el conjunto. Este elemento se acopla con los contrapesos del cigüeñal y ensamblado con la biela y el cojinete. (Funes, 2010)

Figura 3. Muñequilla del cigüeñal 


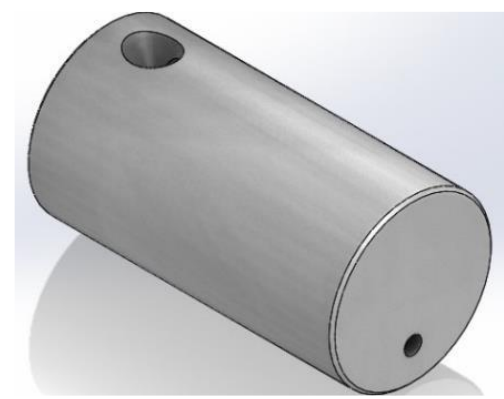

Al realizar el ensamble del conjunto cigüeñal - biela, se tomó en cuenta las respectivas restricciones como es la coincidencia entre planos, operaciones concéntricas y tangenciales, manteniendo una orientación correcta de las partes y piezas, evitando conflictos geométricos y mecánicos. (Lewis Roland W, 2004)

Figura 4. Ensamble del conjunto cigüeñal - biela

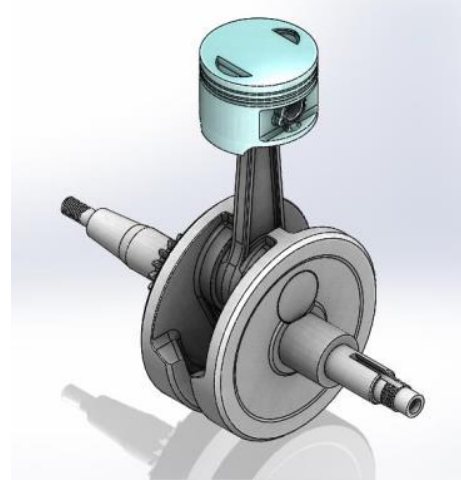

Las condiciones de frontera y el material de los diferentes elementos del conjunto cigüeñal biela que se asignó para el análisis en el software CAE, fueron creados ya que en la base de datos no existe el Acero AISI 1050 y AISI 5120. Se ingreso las características de estos materiales. (MAHMUD, Suhail : WISSAM, Mohamad., 2013)

Se realizó un mallado del mecanismo enfocado a que se comporte de forma real bajo las condiciones asignadas. Este debe ser uniforme en todas las superficies de los elementos. (Barbieri, Bruno, Luchi, \& Rotini, 2009)

Figura 5. Mallado del conjunto cigüeñal - biela 


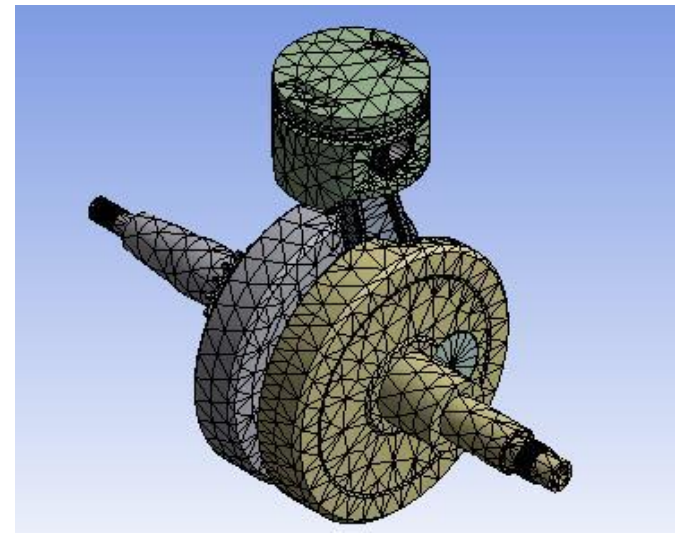

En el análisis de esfuerzos se basó en la teoría de elementos finitos para estructuras y la teoría de volúmenes dinitos para liquidos. Para el mecanismo cigüeñal - biela se aplicó la fuerza media efectiva que fue calculada y datos generales obtenidos del catalogo de la motocicleta. (STOLARSKI, 2006)

Figura 6. Fuerza aplicada en la cabeza del pistón

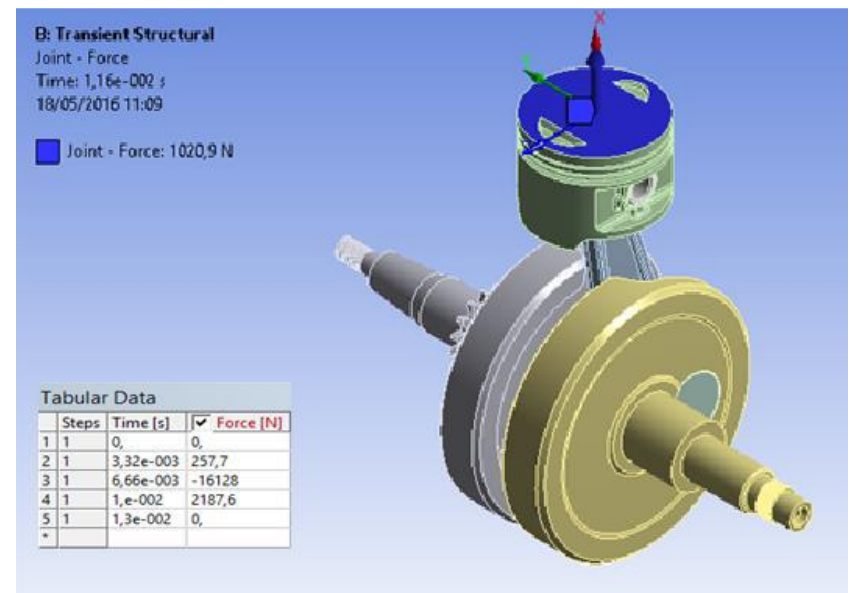

\section{Resultados}

Según el análisis metalográfico se ha podido determinar los materiales de construcción de los elementos del conjunto cigüeñal - biela. En la tabla 1 se expone un resumen de los materiales que son aceros de bajo y medio carbono. (ASKELAND,D., 1998)

Tabla 1. Materiales de los elementos del conjunto cigüeñal - biela

CONJUNTO CIGÜEÑAL - BIELA

ELEMENTO MATERIAL




\section{Contrapesa uno AISI 1050 \\ Contrapesa dos AISI 1050 \\ Biela $\quad$ AISI 5120}

Muñequilla $\quad$ AISI 5120

Elaborado por: Grupo de Investigación

\section{Análisis microestructural contrapesa y eje}

Se diferencian dos microestructuras. En la contrapesa es martensita revenida, mientras que en el eje del cigüeñal está conformada por dos microestructuras en la parte central es Perlita y Ferrita y en su borde es martensita revenida. (Pérez , 2010)

Figura 7. Microestructura de la contrapesa

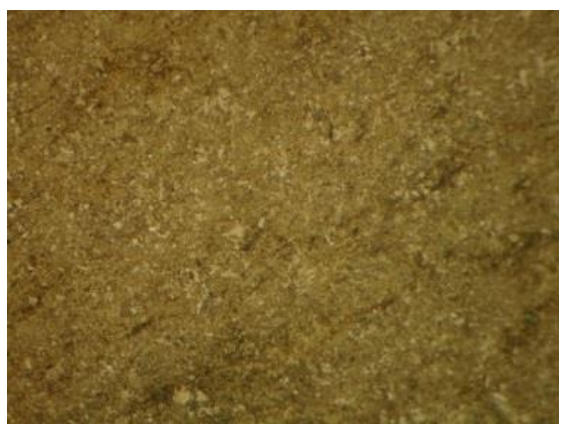

Figura 8. Microestructura del eje del cigüeñal resaltando el cambio de fases

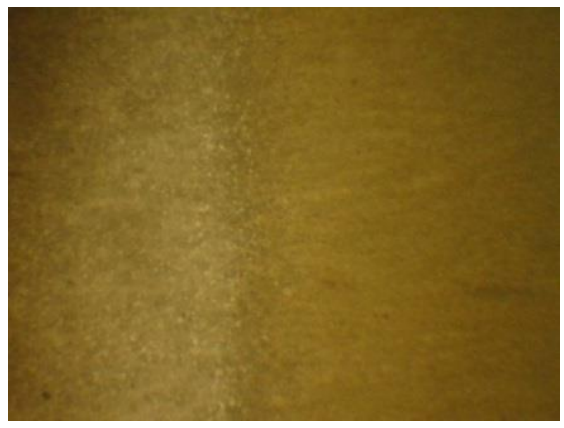

\section{Dureza de los elementos}

Se determinó la dureza en la contrapesa en la escala Rockwell B y en el eje del cigüeñal en la escala Rocwell C. (ASKELAND,D., 1998)

Tabla 2. Dureza de las partes de la contrapesa 


\begin{tabular}{lll}
\hline DUREZA & & \\
\hline ELEMENTO & ESCAL & RESULTAD \\
& A & O \\
Contrapesa & B & $63 \mathrm{HRB}$ \\
Centro del eje & $\mathrm{C}$ & $30 \mathrm{HRC}$ \\
Borde del eje & $\mathrm{C}$ & $50 \mathrm{HRC}$ \\
\hline
\end{tabular}

Elaborado por: Grupo de Investigación

\section{Espectrometría de la contrapesa}

Los datos más relevantes en la composición química de la contrapesa y del eje se encuentra en porcentajes de: Carbono 0,50, Silicio 0,22, Manganeso 0,68 y Cromo 0,15.

\section{Análisis microestructural de la biela}

En la biela se puede observar una composición de martensita revenida.

Figura 9. Microestructura de la biela

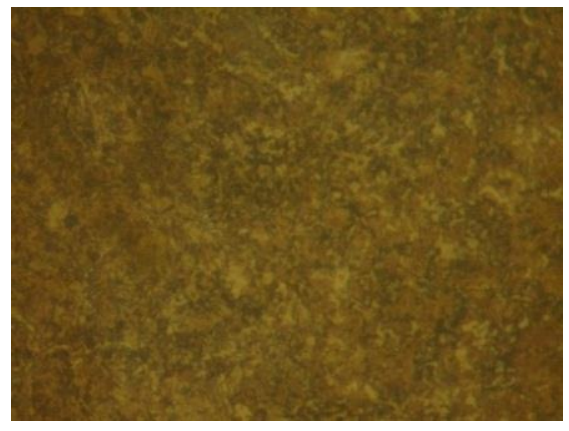

Se determinó que la dureza en la biela es de 41 unidades en la escala Rockwell C.

En la espectrometría para la biela se encuentra la composición química en porcentajes de: Carbono 0,17, Silicio 0,24, Manganeso 0,63 y Cromo 0,95.

\section{Análisis microestructural de la muñequilla}

El análisis microestructura de la muñequilla del cigüeñal determina que está compuesta por martensita revenida.

Figura 10. Microestructura de la muñequilla del cigüeñal 


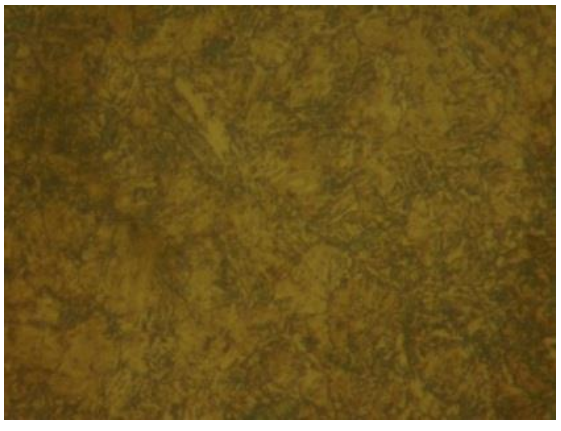

Se determinó que la dureza en la muñequilla es de 50 unidades en la escala Rockwell C.

En la espectrometría para la muñequilla del cigüeñal se encuentra la composición química en porcentajes de: Carbono 0.17, Silicio 0.62, Manganeso 0.62 y Cromo 0.96.

\section{Análisis de esfuerzos}

\section{Análisis Termodinámico del ciclo Otto.}

Se realizará el análisis termodinámico del motor de una motocicleta mono cilíndrica de cuatro tiempos, teniendo como datos las dimensiones del cilindro. (Aparicio, Vera, \& Días, 2001)

Datos:

$\mathrm{I}=1$

$\mathrm{D}=0,057 \mathrm{~m}$

$\mathrm{L}=0,0489 \mathrm{~m}$

$\mathrm{Z}=4$

$T_{1}=80{ }^{\circ} \mathrm{C}$

$P_{1}=101 \mathrm{kPa}$

$\tau=2,8$

$\mathrm{K}=1,4$

$\varphi=9,2: 1$

Desde las dimensiones del cilindro se debe calcular la cilindrada parcial.

$\mathrm{V}_{\mathrm{p}}=\frac{\pi}{4}\left(\mathrm{~d}^{2}\right)(\mathrm{l})$

$$
V_{p}=0,124 \times 10^{-3} \mathrm{~m}^{3}
$$


El mismo que multiplicamos por el número de cilindros y da como resultado la cilindrada total.

$\mathrm{V}_{\mathrm{t}}=\mathrm{Vp}(\mathrm{i})$

$\mathrm{V}_{\mathrm{t}}=0,124 \times 10^{-3} \mathrm{~m}^{3}$

La relación de compresión en los motores a gasolina varía de 6 a 10, donde se ha escogido una relación de 9,5. A partir de la relación de compresión se obtiene el volumen uno o volumen total del cilindro. (Francisco Payri, 2011)

$\varphi=\frac{\mathrm{V}_{1}}{\mathrm{~V}_{\mathrm{c}}}$

$\mathrm{V}_{\mathrm{t}}=\frac{\varphi\left(\mathrm{V}_{\mathrm{p}}\right)}{\varphi-1}$

$V_{1}=0,14 \times 10^{-3} \mathrm{~m}^{3}$

Volumen de la cámara $\left(\mathrm{V}_{\mathrm{c}}=\mathrm{V}_{2}\right)$

$\mathrm{V}_{1}=\mathrm{V}_{2}+\mathrm{V}_{\mathrm{p}}$

$V_{2}=0,016 \times 10^{-3} \mathrm{~m}^{3}$

\section{Proceso Adiabático Isentrópico de Compresión.}

$$
\begin{aligned}
& \frac{\mathrm{V}_{1}}{\mathrm{~V}_{2}}=\left(\frac{\mathrm{P}_{2}}{\mathrm{P}_{1}}\right)^{\left(\frac{1}{\mathrm{k}}\right)} \\
& \mathrm{P}_{2}=\left(\frac{\mathrm{v}_{1}}{\mathrm{v}_{2}}\right)^{\left(\frac{1}{\mathrm{k}}\right)}\left(\mathrm{P}_{1}\right)
\end{aligned}
$$

$$
\mathrm{P}_{2}=2,38 \mathrm{MPa}
$$

Al tener la temperatura uno, que es la temperatura a la que trabaja el motor en condiciones normales, se puede determinar la temperatura dos. (KINDLER, 1986)

$\mathrm{T}_{2}=\mathrm{T}_{1}(\varphi)^{\mathrm{K}-1}$

$$
\begin{gathered}
\mathrm{T}_{2}=194,35^{\circ} \mathrm{C} \\
\mathrm{V}_{2}=\mathrm{V}_{3}=0,27 \times 10^{-3} \mathrm{~m}^{3}
\end{gathered}
$$


Proceso de suministro de calor a $\mathrm{V}=$ cte. La relación de compresión a volumen contante (grado de elevación de la presión) puede variar de 2,8 a 4,2, por lo que se ha escogido un valor de 2,8 para poder sacar la presión 3 y la temperatura 3. (Borja Martínez \& Navas Gallegos, 2013)

$P_{3}=\tau\left(P_{2}\right)$

$$
\mathrm{P}_{3}=6,3 \mathrm{MPa}
$$

$\frac{P_{2}}{T_{2}}=\frac{P_{3}}{T_{3}}$

$\mathrm{T}_{3}=\frac{\mathrm{P}_{3}\left(\mathrm{~T}_{2}\right)}{\mathrm{P}_{2}}$

$$
\mathrm{T}_{3}=544,2^{\circ} \mathrm{C}
$$

Proceso adiabático Isentrópico de expansión.

$\mathrm{T}_{4}=\frac{\mathrm{T}_{3}}{(\varphi)^{(\mathrm{k}-1)}}$

$\mathrm{T}_{4}=224^{\circ} \mathrm{C}$

$\mathrm{V}_{1}=\mathrm{V}_{4}$

$\mathrm{P}_{4}=\left[\frac{\mathrm{P}_{3}\left(\mathrm{~V}_{3}^{\mathrm{k}}\right)}{\mathrm{V}_{4}}\right]^{\left(\frac{1}{\mathrm{~K}}\right)}$

$\mathrm{P}_{4}=856,9 \mathrm{kPa}$

Una vez obtenidas todas presiones en cada ciclo del motor, calculamos las fuerzas.

$A=\frac{\pi\left(d^{2}\right)}{4}$

$$
A=2,55 \times 10^{-3} \mathrm{~m}^{2}
$$

$\mathrm{P}=\frac{\mathrm{F}}{\mathrm{A}}$

$\mathrm{F}_{1}=\mathrm{P}_{1}(\mathrm{~A})$

$$
\mathrm{F}_{1}=257,3 \mathrm{~N}
$$

$\mathrm{F}_{2}=\mathrm{P}_{2}(\mathrm{~A})$

$$
\mathrm{F}_{2}=5754,16 \mathrm{~N}
$$


Calcular la fuerza máxima a la que está sometido el pistón.

$\mathrm{F}_{3}=\mathrm{P}_{3}(\mathrm{~A})$

$$
\mathrm{F}_{3}=16128,23 \mathrm{~N}
$$

Calcular la fuerza mínima a la que está sometido el pistón.

$$
\mathrm{F}_{4}=\mathrm{P}_{4}(\mathrm{~A})
$$

$$
\mathrm{F}_{4}=2185,3 \mathrm{~N}
$$

\section{Análisis de esfuerzos mediante software.}

Aquí se puede apreciar el esfuerzo máximo y mínimo de $1,1 \times 10^{8} \mathrm{~Pa}$ y $57,69 \mathrm{~Pa}$ correspondientemente. Mediante un código de colores se puede diferenciar los resultados de las cargas a los que está sometido el conjunto. El color azul que recubre las diferentes superficies significa que están soportando cargas mínimas, mientras que las superficies que son recubiertas por diferentes colores hasta llegar al color rojo significa que están soportando mayores cargas hasta llegar a la carga máxima. (Rao , 2005)

Figura 11. Esfuerzo máximo del conjunto cigüeñal - biela

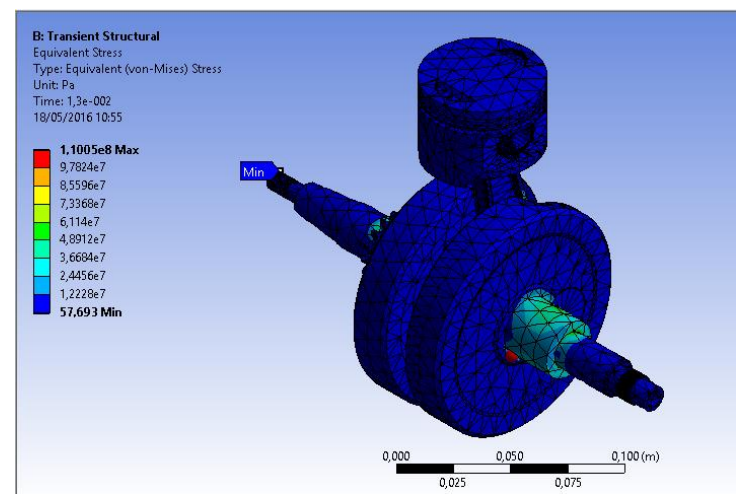

Se observa los resultados mediante un código de colores. El color rojo que recubre las superficies del conjunto nos indica que no puede soportar más cargas de las aplicadas anteriormente con un valor de 3,36 como mínimo y como máximo 15, el color azul que recubre la mayor parte de las piezas nos indica que pueden soportar las cargas mencionadas y también cargas adicionales. Con estos valores podemos determinar que el conjunto puede soportar mayores revoluciones y mayores esfuerzos. (STOLARSKI, 2006)

Figura 12. Coeficiente de seguridad del conjunto cigüeñal - biela 


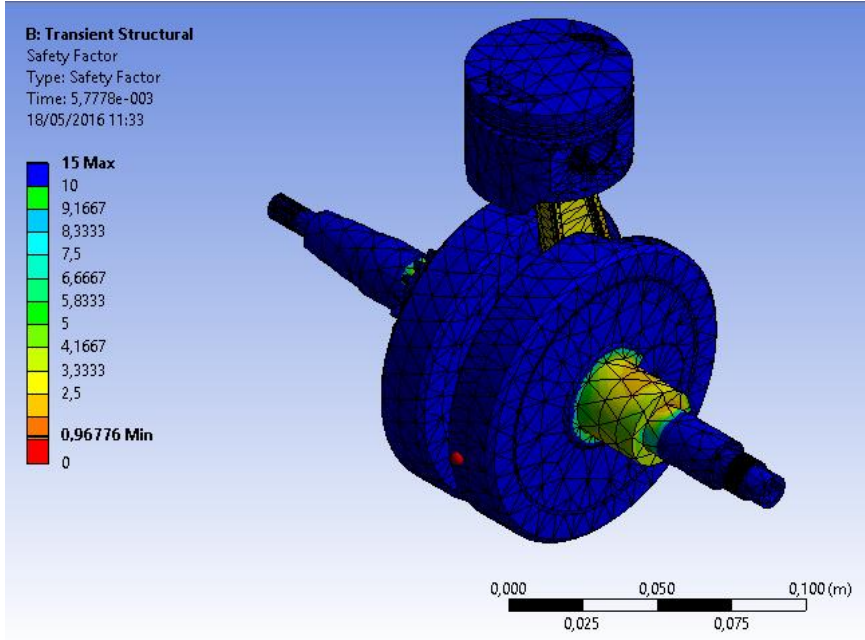

\section{Discusión}

En este proyecto se realizó el estudio de cada una de las piezas del conjunto cigüeñal-biela, en donde se determinó que los materiales de dichas piezas son de acero de medio y bajo carbono, estos aceros no se producen dentro del país. En el caso de piezas sinterizadas el costo de fabricación seria alto por el requerimiento de maquinaria especializada. (Conesa, 2011)

En la espectrometría para el cigüeñal se encuentra la composición química porcentajes de: Carbono 0,50, Silicio 0,22, Manganeso 0,68 y Cromo 0,15. Se determinó la dureza en la contrapesa de 63 unidades en la escala Rockwell B y en el eje del cigüeñal la dureza es de 50 unidades en la escala Rocwell C. El análisis microestructural se observa que el cigüeñal a pesar de ser del mismo material se diferencia por sus tratamientos térmicos, en la contrapesa y en el eje. La contrapesa del cigüeñal tiene una microestructura de martensita revenida, mientras que en el eje del cigüeñal está conformada por Perlita y Ferrita.

Las pruebas que se realizaron al cigüeñal determinan que el material es un acero AISI 1050, pero dicho material no es comercial dentro del Ecuador. En la espectrometría para la biela se encuentra la composición química en porcentajes de: Carbono 0,17, Silicio 0,24, Manganeso 0,63 y Cromo 0,95 .

Se determinó que la dureza en la biela es de 41 unidades en la escala Rockwell C. El análisis microestructural de la biela se determina que está compuesta por martensita revenida. En la espectrometría para la muñequilla del cigüeñal se encuentra la composición química en porcentajes de: Carbono 0.17, Silicio 0.62, Manganeso 0.62 y Cromo 0.96. Por lo que se determina que es un acero AISI 5120 de bajo carbono. 
Se determinó que la dureza en la muñequilla del cigüeñal es de 50 unidades en la escala Rockwell C. El análisis microestructura de la muñequilla del cigüeñal determina que está compuesta por martensita revenida.

En las pruebas realizadas, se determinó que el material de la biela y de la muñequilla del cigüeñal es un Acero AISI 5120, pero dicho material no es comercial dentro del Ecuador.

\section{Conclusiones.}

- Los aceros AISI 1050 Y AISI 5120, son materiales que se utilizan para la fabricación específicamente de ejes, cigüeñales y bielas, en el Ecuador no existen fábricas de dichas partes y piezas, razón por la cual no hay la necesidad de comerciarlo dentro del país.

- Se ha realizado el análisis del conjunto cigüeñal - biela en el Software ANSYS, en donde se ha obtenido un esfuerzo máximo de $1,895 \times 10^{7} \mathrm{~Pa}$ y un mínimo de 28,74 $\mathrm{Pa}$, y un coeficiente de seguridad máximo de 15 y mínimo de 1,035 a partir de dichos datos se determina que el esfuerzo máximo y el coeficiente de seguridad al que está sometido dicho conjunto están dentro de los parámetros permisibles para el diseño.

- El cálculo termodinámico determina que la fuerza mínima es de 257,71 N, fuerza máxima de $16128,23 \mathrm{~N}$ y la fuerza media efectiva de $6402,01 \mathrm{~N}$.

\section{Referencias bibliográficas.}

Aparicio, F., Vera, C., \& Días, V. (2001). Teoría de los vehículos automóviles (Segunda ed.). Madrid, España: Sección de publicaciones de la Escuela Técnica Superior de Ingenieros Industriales.

Askeland, D. (1998). Ciencia e Ingenieria de materiales. Mexico: International Thomson.

Barbieri, L., Bruno, F., Luchi, M., \& Rotini, F. (2009). Design of automotive components using advanced CAE systems. 21st European Modeling and Simulation Symposium.

Bazela, C. (2012). moto.com.br. Recuperado el 2015, de http://www.moto.com.br/acontece/conteudo/moto_guzzi_v7_tres_versoes_em_um_ mesmo_modelo-49849.html

Borja Martínez, L., \& Navas Gallegos, R. (2013). Eficiencia y transformación energética: modificación de una motocicleta de combustión interna a energía eléctrica. (Bachelor's thesis, Universidad San Francisco de quito). Quito.

Conesa, J. (2011). Motor de Combustión Interna.

Francisco Payri, J. M. (2011). Motores de Combustión Interna Alternativos (Primera ed., Vol. I). España: Reverte.

Fuentes, L. (2011). El método de los elementos finitos. (Bachelor's thesis, Univwersidad de Salamanca).

Funes, C. A. (2010). Motores para Competición: Diseño y Preparación (Primera ed., Vol. 1). Córdova, Córdova, Argentina. 
Hernández Sampieri, R., Fernández Collado, C., \& Baptista Lucio, P. (2010). Metodología de la investigación.

Kindler, H. (1986). Matemática aplicada para la técnica del automóvil (Octava ed.). (J. Company, Trad.) Barcelona, España: Reverté.

Lewis Roland W, N. P. (2004). Fundamentals of the Finite Element Method for Heat and Fluid Flow. United Kindong: Wiley.

Mahmud, Suhail : WISSAM, Mohamad. (2013). Introduction to Ansys Workbench. Emiratos Arabes: Aviation College. Retrieved from http://inside.mines.edu/ apetrell/ENME442/Labs/1301_ENME442_lab2.1.pdf

Martínez, D. H. (s.f.). Manual Practico del Automóvil, Reparación, Mantenimiento y Prácticas.

Pérez , V. (2010). Evaluación no destructiva de materiales mediante métodos de reconstrucción tomográfica basados en redes neuronales de base radial. España.

Rao , S. (2005). The Finite Element Method in Engineering (Vol. Cuarta Edición). EEUU.: Elsevier Inc. doi:978-0-7506-7828-5

Rovira, J., \& Muñoz, M. (2015). Motores de combustión interna. UNED.

SolidWorks Corporation. (2017). SolidWorks. (D. Systemes, Editor) Retrieved from https://www.solidworks.es/sw/products/simulation/finite-element-analysis.htm

Stolarski, T. :. (2006). Engineering analysis with ansys software. Amsterdam: Elsevier.

Suzuki Motor de Colombia. (17 de Enero de 2016). www.suzuki.com.co. Obtenido de www.suzuki.com.co: https://www.suzuki.com.co/motocicletas/gn-125-326

Villena Izurieta, N. P. (2015). El Ecuador y el proceso de cambio de la matriz productiva: consideraciones para el desarrollo y equilibrio de la balanza comercial. Observatorio de la Economía Latinoamericana.

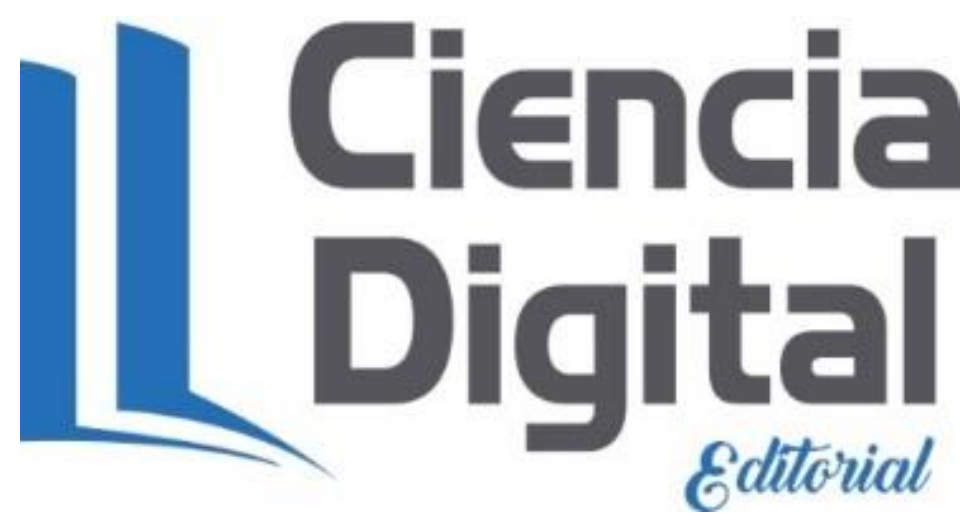




\section{Para citar el artículo indexado.}

Padilla C, Cuaical B. \& Buenaño L. (2019). Análisis microestructural y diseño de cigüeñal y biela de un motor mono cilíndrico de 4 tiempos. Revista electrónica Ciencia Digital 3(1), 298-312. Recuperado desde: http://cienciadigital.org/revistacienciadigital2/index.php/CienciaDigital/article/view/293/69 7

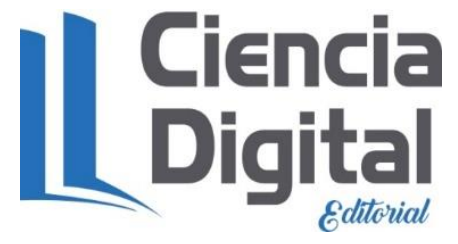

El artículo que se publica es de exclusiva responsabilidad de los autores y no necesariamente reflejan el pensamiento de la Revista Ciencia Digital.

El artículo queda en propiedad de la revista y, por tanto, su publicación parcial y/o total en otro medio tiene que ser autorizado por el director de la Revista Ciencia Digital.
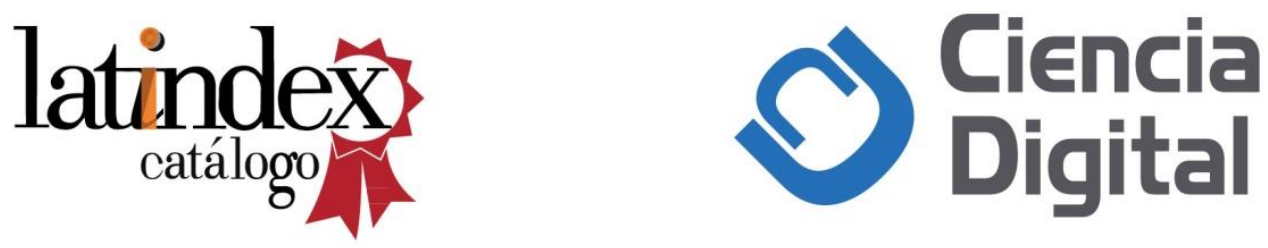